\title{
Bevacizumab Efficacy in Metastatic Colorectal Cancer is Dependent on Primary Tumor Resection
}

\author{
Francois Ghiringhelli, MD, $\mathrm{PhD}^{1,2}$, Damien Bichard, PharmD $^{3,4}$, Samuel Limat, PharmD $^{3,4}$, Veronique Lorgis, \\ $\mathrm{MD}^{1}$, Julie Vincent, $\mathrm{MD}, \mathrm{PhD}^{1,2}$, Christophe Borg, $\mathrm{MD}, \mathrm{PhD}^{3,5,6}$, Julie Berthou, PharmD ${ }^{3}$, David Orry, $\mathrm{MD}^{7}$, \\ Pablo Ortega-Deballon, MD, PhD ${ }^{2,8}$, Zaher Lakkis, $\mathrm{MD}^{9}$, Olivier Facy, MD, PhD ${ }^{2,8}$, Bruno Heyd, $\mathrm{MD}^{9}$, Patrick Rat, \\ $\mathrm{MD}^{2,8}$, Virginie Nerich, PharmD ${ }^{3,4}$, and Sylvain Ladoire, $\mathrm{MD}, \mathbf{P h D}^{1,2}$ \\ ${ }^{1}$ Department of Medical Oncology, Centre Georges-François Leclerc, INSERM Avenir 866, Dijon, France; ${ }^{2}$ Institut \\ National de la Santé et de la Recherche Médicale (INSERM), UMR-866, University of Burgundy, Dijon, France; ${ }^{3}$ Institut \\ National de la Santé et de la Recherche Médicale (INSERM), UMR-1098, Besançon, France; ${ }^{4}$ Department of Pharmacy, \\ University Hospital of Besançon, Besançon, France; ${ }^{5}$ Institut National de la Santé et de la Recherche Médicale (INSERM), \\ UMR 1098, University of Franche Comté, Besançon, France; ${ }^{6}$ Department of Medical Oncology, University Hospital of \\ Besançon, Besançon, France; ${ }^{7}$ Department of Oncologic Surgery, Centre Georges-François Leclerc, Dijon, France; \\ ${ }^{8}$ Department of Digestive and Oncologic Surgery, University Hospital of Dijon, Dijon, France; ${ }^{9}$ Department of Digestive \\ and Oncologic Surgery, University Hospital of Besançon, Besançon, France
}

\begin{abstract}
Purpose. Bevacizumab plus fluoropyrimidine-based chemotherapy is standard treatment for first-line and secondline metastatic colorectal cancer (mCRC). However, to date, there is no current biomarker predictive for the benefit of bevacizumab use for these patients. Preclinical data suggest that the presence of the primary tumor could be involved in less efficient antitumor activity of antiangiogenic agents, but no clinical data currently support this hypothesis.

Methods. We performed a retrospective analysis of factors associated with overall survival (OS) in a study cohort of 409 mCRC patients. Univariate and multivariate Cox proportional hazard regression models were used to assess the influence of primary tumor resection and bevacizumab use on OS. We evaluated associations linking bevacizumab use and OS
\end{abstract}

Electronic supplementary material The online version of this article (doi:10.1245/s10434-013-3463-y) contains supplementary material, which is available to authorized users.

(C) The Author(s) 2014. This article is published with open access at Springerlink.com

First Received: 8 October 2013;

Published Online: 14 January 2014

F. Ghiringhelli, MD, PhD

e-mail: francois.ghiringhelli@yahoo.fr; fghiringhelli@cgfl.fr

S. Ladoire, $\mathrm{MD}, \mathrm{PhD}$

e-mail: sladoire@cgfl.fr among patients who previously underwent or did not undergo primary tumor resection. Results were externally validated in a second independent cohort of $328 \mathrm{mCRC}$ patients.

Results. In the study cohort, bevacizumab use and resection of the primary tumor were associated with improved OS. However, subgroup analyses indicate that bevacizumab did not influence survival of patients bearing a primary colorectal tumor (hazard ratio (HR) 0.98, $95 \%$ confidence interval (CI) $0.60-1.61, \log$-rank test $P=0.6$ ). By contrast, the survival benefit of bevacizumab was restricted to patients who previously underwent primary tumor resection (HR 0.71, $95 \%$ CI 0.55-0.92, $P=0.009$ ). Similar results were observed in the validation cohort.

Conclusions. Addition of bevacizumab to chemotherapy is associated with improvement of OS only in patients with primary tumor resection. These data support the rationale to validate prospectively the influence of primary tumor resection on bevacizumab antitumor effect in synchronous mCRC.

There are no curative options in patients with unresectable metastases from colorectal cancer (mCRC), but treatment with systemic chemotherapy improves overall survival (OS). ${ }^{1}$ Therapeutic options currently available rely on three cytotoxic chemotherapies, fluoropyrimidine, oxaliplatin, and irinotecan, associated with targeted-therapy anti-epidermal growth factor receptor (EGFR) or anti-vascular endothelial growth factor (VEGF). The 5-year OS in patients who are diagnosed with 
unresectable distant metastases ranges between 10 and $20 \% .^{2-4}$ In contrast, when metastases can be surgically removed, 5-year OS increases to $20-50 \% .^{5}$ Recent clinical studies suggest that survival continues to improve with the routine addition of targeted therapies. ${ }^{6-8}$ For instance, phase III trials have shown that adding bevacizumab to first- or secondline chemotherapy may modestly but significantly improve mCRC patient survival. Moreover, the continued use of bevacizumab beyond disease progression leads to a significant improvement in OS and progression-free survival compared with postprogression chemotherapy alone..$^{6,8-11}$ The association between survival improvement and bevacizumab addition to routine chemotherapy regimens has been also confirmed in large populations of patients with $\mathrm{mCRC}$ in prospective cohorts in the context of general oncology practice. ${ }^{12,13}$ Consequently, VEGF inhibition has become an attractive therapeutic target in patients with $\mathrm{mCRC}$; however, there is no current predictive biomarker for the efficacy and the clinical benefit of bevacizumab in terms of survival improvement in mCRC patients. This becomes an important goal for clinical trial design and should permit their more rational use in patients with mCRC.

Recent preclinical data have demonstrated that molecular mechanisms involved in neoangiogenesis are different in the primary tumor compared to distant metastases. ${ }^{14}$ Furthermore, in nonresected primary tumor animal models, angiogenesis inhibitors targeting the VEGF pathway demonstrated antitumor effects but concomitantly elicited more invasive or metastatic behavior. ${ }^{15}$

Thus, these data led us to hypothesize that the presence of primary tumor may negatively affect the efficacy of antiVEGF targeted therapy, leading to lower clinical efficacy. To answer this question, we designed a retrospective clinical study to assess whether the resection of the primary tumor before starting chemotherapy could be associated with patient outcome in mCRC patients receiving chemotherapy with or without bevacizumab.

\section{PATIENTS AND METHODS}

\section{Patients}

From January 2001 to December 2011, 409 consecutive patients with histologically proven metastatic colorectal adenocarcinoma received first-line chemotherapy treatment at the Georges-François Leclerc Cancer Center (Dijon, France) and were prospectively recorded in an institutional clinical database, which made them eligible for this retrospective study. As first- or second-line treatment, 233 patients were provided chemotherapy plus bevacizumab (bevacizumab group), while 176 patients received chemotherapy alone without bevacizumab (chemotherapy-alone group). Forty- three patients were included in clinical trials for first- or second-line chemotherapy for metastatic disease involving the use of bevacizumab or cetuximab. This $\mathrm{mCRC}$ patient cohort constituted the study cohort. The results obtained for this cohort were validated in an independent validation cohort of 328 consecutive mCRC patients who were treated at Besancon University Hospital from 1997 to 2009.

This retrospective study was approved by our Institutional Review Board, and all data were anonymized. Patients received bevacizumab plus fluoropyrimidinebased chemotherapy (chosen at the clinician's discretion: single-agent fluoropyrimidine or fluoropyrimidine plus oxaliplatin or irinotecan) until disease progression. The bevacizumab dose was $5 \mathrm{mg} / \mathrm{kg}$ every 2 weeks (5-fluorouracil-based regimens) or $7.5 \mathrm{mg} / \mathrm{kg}$ every 3 weeks (capecitabine-based regimens). Bevacizumab was administered intravenously, initially over $90 \mathrm{~min}$. If the first infusion was well tolerated, the second was delivered over $60 \mathrm{~min}$; if the 60-min infusion was well tolerated, all subsequent infusions were delivered over $30 \mathrm{~min}$.

\section{Statistical Analysis}

All patients were followed up until death or the date of the end of the study (December 31, 2011). The primary end point was OS, which was defined as the interval between date of first dose of chemotherapy and date of death as reported on medical record or December 31, 2011, whichever occurred first. Survivors were censored at last follow-up.

Median follow-up with its $95 \%$ confidence interval (CI) was calculated by the reverse Kaplan-Meier method. The association between the treatment groups (bevacizumab or chemotherapy alone) and other patient and disease characteristics were examined by the Chi-square test and Fisher's exact probability test, or the Mann-Whitney test if required.

The Kaplan-Meier estimation was used for calculation of survival probabilities and the log-rank test for comparison of survival curves. Cox proportional hazard regression was used to estimate the hazard ratio (HR) and $95 \% \mathrm{CI}$ for univariate and multivariate analysis of OS. Classical prognostic factors in mCRC were systematically examined in analyses and included bevacizumab use, anti-EGFR therapy use, number of metastatic sites, age (continuous), sex, serum carcinoembryonic antigen (CEA) level determined before the first injection of chemotherapy (continuous), B-Raf and K-Ras status, World Health Organization (WHO) performance status, presence of synchronous or metachronous disease, complete resection of all metastases, and primary tumor resection before the beginning of chemotherapy for metastatic disease. No information of the measure of the bulk of the disease was collected. 
TABLE 1 Patient and tumor characteristics $(n=409)$

\begin{tabular}{|c|c|c|c|c|c|}
\hline Characteristic & Variable & Chemotherapy alone $(n=175)$ & $\begin{array}{l}\text { Bevacizumab } \\
(n=234)\end{array}$ & $\begin{array}{l}\text { Overall } \\
(n=409)\end{array}$ & $P$ value \\
\hline \multirow[t]{2}{*}{ Age (year) } & Median [min; $\max ]$ & $66.8[43 ; 88]$ & $63.5[24 ; 90]$ & $65.5[24 ; 90]$ & 0.06 \\
\hline & Mean (SD) & $66.2(10.3)$ & $64(11.5)$ & $65(11)$ & \\
\hline \multirow[t]{2}{*}{ Sex } & Male & $99(56 \%)$ & $120(51 \%)$ & $219(53.5 \%)$ & 0.33 \\
\hline & Female & $76(44 \%)$ & $114(49 \%)$ & $190(46.5 \%)$ & \\
\hline Death & & $154(88 \%)$ & $158(67.5 \%)$ & 312 & $<0.0001$ \\
\hline \multirow[t]{5}{*}{ WHO PS } & 0 & $54(31 \%)$ & $83(35 \%)$ & $137(34 \%)$ & 0.06 \\
\hline & 1 & $47(27 \%)$ & $58(25 \%)$ & $105(25 \%)$ & \\
\hline & 2 & $19(11 \%)$ & $15(6 \%)$ & $34(8 \%)$ & \\
\hline & 3 & $7(4 \%)$ & $2(0.5 \%)$ & $9(2 \%)$ & \\
\hline & Unknown & $48(27 \%)$ & $81(33.5 \%)$ & $129(31 \%)$ & \\
\hline \multirow[t]{3}{*}{ B-Raf status } & Wild type & $13(7 \%)$ & $93(40 \%)$ & $106(26 \%)$ & 0.9 \\
\hline & Mutated & $1(1 \%)$ & $11(5 \%)$ & $12(3 \%)$ & \\
\hline & Unknown & $161(92 \%)$ & $130(55 \%)$ & $291(71 \%)$ & \\
\hline \multirow[t]{3}{*}{ K-Ras status } & Wild type & $13(7 \%)$ & $100(43 \%)$ & $113(27 \%)$ & 0.92 \\
\hline & Mutated & $9(5 \%)$ & $80(34 \%)$ & $89(22 \%)$ & \\
\hline & Unknown & $153(88 \%)$ & $54(23 \%)$ & $207(51 \%)$ & \\
\hline \multirow[t]{2}{*}{ Evolution } & Synchronous & $94(54 \%)$ & $158(67.5 \%)$ & $252(62 \%)$ & 0.004 \\
\hline & Metachronous & $81(46 \%)$ & $76(32.5 \%)$ & $157(38 \%)$ & \\
\hline \multirow[t]{2}{*}{ Primary tumor resection } & Yes & $140(80 \%)$ & $193(82.5 \%)$ & $333(81.5 \%)$ & 0.47 \\
\hline & No & $35(20 \%)$ & $41(17.5 \%)$ & $76(18.5 \%)$ & \\
\hline \multirow[t]{2}{*}{ Complete surgery of metastases } & No & $137(78 \%)$ & $167(71 \%)$ & $304(74 \%)$ & 0.12 \\
\hline & Yes & $38(22 \%)$ & $67(29 \%)$ & $105(26 \%)$ & \\
\hline \multirow[t]{3}{*}{ Localization of the primary tumor } & Colon & $136(78 \%)$ & $184(78.5 \%)$ & $320(78 \%)$ & 0.79 \\
\hline & Rectum & $39(22 \%)$ & $48(20.5 \%)$ & $87(21.5 \%)$ & \\
\hline & Unknown & 0 & $2(1 \%)$ & $2(0.5 \%)$ & \\
\hline \multirow[t]{2}{*}{ EGFR therapy } & Yes & $63(36 \%)$ & $104(44.5 \%)$ & $167(41 \%)$ & 0.11 \\
\hline & No & $112(64 \%)$ & $130(55.5 \%)$ & $242(59 \%)$ & \\
\hline \multirow[t]{3}{*}{ No. of treatment lines } & 1 & $32(18 \%)$ & $19(8 \%)$ & $51(12.5 \%)$ & $<\mathbf{0 . 0 0 0 1}$ \\
\hline & 2 & $41(23.5 \%)$ & $30(13 \%)$ & $71(17 \%)$ & \\
\hline & 3 or more & $102(58.5 \%)$ & $185(79 \%)$ & $287(70.5 \%)$ & \\
\hline \multirow[t]{2}{*}{ No. of metastatic sites } & 1 & $110(63 \%)$ & $138(59 \%)$ & $248(60 \%)$ & 0.48 \\
\hline & $>1$ & $65(38 \%)$ & $96(41 \%)$ & $161(40 \%)$ & \\
\hline \multirow[t]{2}{*}{ CEA level } & Median [min; $\max ]$ & $17[0 ; 20,800]$ & $18[0 ; 14,660]$ & $18[0 ; 20,800]$ & 0.6 \\
\hline & Mean (SD) & $540(2,405)$ & $380(1,440)$ & $447(1,961)$ & \\
\hline
\end{tabular}

WHO World Health Organization, $P S$ performance status, EGFR epidermal growth factor receptor, CEA carcinoembryonic antigen

All predictors with $P$ values lower than 0.05 in univariate analysis were used in multivariate analysis (except variables with more than $25 \%$ of missing data). Correlations between co-variables were first tested for eligible variables. To prevent collinearity, when two variables were significantly correlated, one variable was retained according to its clinical relevance or to the value of the likelihood ratio (e.g., evolution and primary tumor resection). All analyses were performed by Stata software, version 11 (StataCorp, College Station, TX). $P$ values were two-tailed and were considered significant when less than 0.05 .

\section{RESULTS}

\section{Patients From Study Cohort}

Between January 2001 and December 2011, 409 mCRC patients were treated in Georges Francois Leclerc Cancer Center. Of these, 233 patients (57\%) received bevacizumab during their chemotherapeutic treatment for metastatic disease. Patient and tumor characteristics are listed in Table 1. There was no significant difference between the bevacizumab and chemotherapy-alone groups 


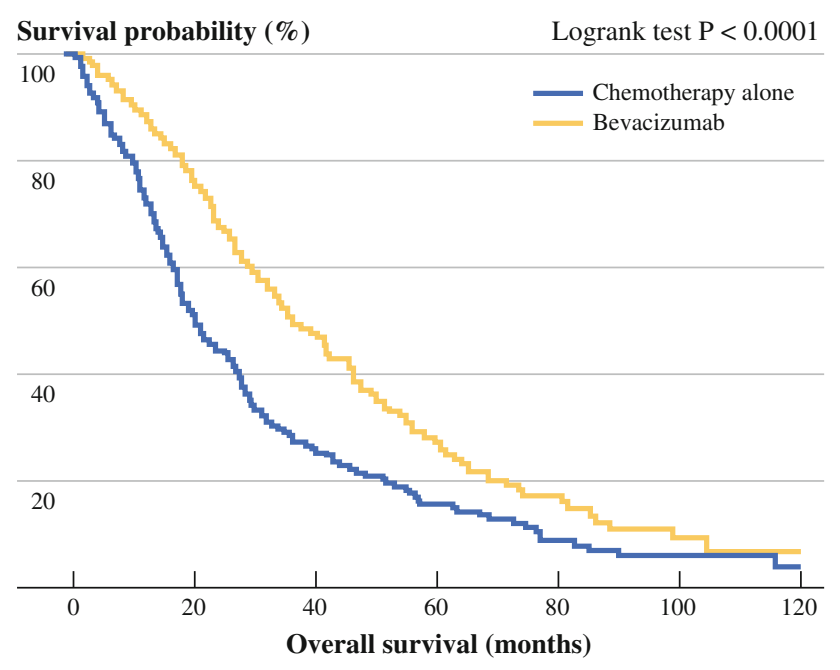

FIG. 1 Kaplan-Meier curve for OS in the study cohort $(n=409)$ of mCRC patients, stratified according to treatment: chemotherapy with bevacizumab (bevacizumab group), or chemotherapy without bevacizumab (chemotherapy-alone group). $P$ value was calculated by log-rank test

for the main clinicobiological characteristics, except for type of metastatic disease (more synchronous metastatic disease in the bevacizumab group: 54 vs. $67.5 \%$, $P=0.004)$ and except for the proportion of patients who received 3 or more chemotherapeutic lines, which was significantly higher in the bevacizumab group (79 vs. $58.5 \%, P<0.0001)$. Median follow-up at the data cutoff point was 31 months in the bevacizumab group and 33 months in the chemotherapy group.

\section{Patients from Validation Cohort}

Between January 1997 and December 2009, 328 mCRC patients were treated at the Besancon University Hospital. Of these, 177 patients (54\%) received bevacizumab during their chemotherapeutic treatment for metastatic disease. Patients and tumor characteristics are provided in Supplementary Table S1. As for the study cohort, there was no significant difference between the bevacizumab and chemotherapy-alone groups for the main available clinicobiological characteristics, except for anti-EGFR treatment, which was more frequently received by patients in the bevacizumab group (53 vs. $26 \%, P<0.0001$ ). Median follow-up at the data cutoff point was 23 months in the bevacizumab group and 17 months in the chemotherapy-alone group.

\section{OS in Bevacizumab and Chemotherapy-alone Groups in the Study Cohort}

Proportions of patients who died were $88 \%$ in the chemotherapy-alone group, and $67.5 \%$ in the bevacizumab group $(P<0.0001)$. Patients receiving bevacizumab had a better outcome in term of OS compared to patients from the chemotherapy-alone group (log-rank test $P<0.0001$ ) (Fig. 1). Median OS was 35.8 months with bevacizumab and 20.1 months without bevacizumab. Univariate analysis indicates that age, high CEA level, WHO performance status $\geq 2$, B-Raf mutated tumor status, synchronous metastatic disease, absence of complete surgery of metastases, localization of primary tumor in colon, absence of antiEGFR therapy, and more than 1 metastatic site were significantly associated with poorer OS (Table 2). Bevacizumab use (HR 0.61, $95 \%$ CI $0.49-0.77$, $P<0.0001$ ) and primary tumor resection (HR $0.32,95 \%$ CI $0.24-0.43, P<0.0001)$ were significantly associated with improved OS (Table 2).

By multivariate analysis, high CEA levels and absence of complete removal of metastases remained independently associated with poorer OS, bevacizumab use (HR 0.64, $95 \%$ CI $0.50-0.81, P<0.0007)$, and primary tumor resection (HR 0.36, $95 \%$ CI $0.26-0.49, P<0.0001$ ) (Table 2).

\section{Effect of Primary Tumor Resection and Bevacizumab Use on OS in the Study Cohort $(n=409)$}

Because our initial hypothesis was that primary tumor resection could affect bevacizumab antitumor efficacy, we performed a subgroup analysis to assess the association of survival improvement with bevacizumab use both in patients who initially underwent primary tumor resection $(n=333)$ and in nonresected patients $(n=76)$. Both groups of patients with or without primary tumor resection were comparable for classical prognostic factors (Table 3). We only noted a significantly higher number of patients who received 3 or more treatment lines in the primary tumor resection group, which was probably linked to the longer survival in these patients and which is a classical problem of immortal time bias. ${ }^{16}$

Survival analysis revealed that the addition of bevacizumab failed to improve mCRC patient's survival when primary tumor was present. In these patients, median OS was 18.5 months for the bevacizumab group and 17.1 months in the chemotherapy-alone group (HR 0.98, $95 \%$ CI $0.60-1.61$, log-rank test $P=0.6$ ) (Fig. 2b). Nevertheless, as previously demonstrated, bevacizumab use significantly improved OS in patients who underwent primary tumor resection before beginning chemotherapy. In these patients with primary tumor resection, median OS was 41.9 months in the bevacizumab group and 25.3 months in the chemotherapy-alone group (HR 0.61, $95 \%$ CI $0.47-0.79$, log-rank test $P=0.0003$ ) (Fig. 2a). By subgroup analysis and multivariate Cox model, we found that bevacizumab remained an independent 
TABLE 2 Univariate and multivariate analysis (Cox regression) for factors associated with overall survival

\begin{tabular}{|c|c|c|c|c|c|c|c|}
\hline \multirow[t]{2}{*}{ Characteristic } & \multirow[t]{2}{*}{ Variable } & \multicolumn{3}{|c|}{ Univariate analysis } & \multicolumn{3}{|c|}{ Multivariate analysis } \\
\hline & & HR & $95 \% \mathrm{CI}$ & $P$ value & HR & $95 \% \mathrm{CI}$ & $P$ value \\
\hline $\mathrm{Age}^{\mathrm{a}}$ & & 1.0137 & $1.0004-1.0272$ & 0.04 & 1.0044 & {$[0.99 ; 1.02]$} & 0.54 \\
\hline \multirow[t]{2}{*}{ Sex } & Male & 1 & & & & & \\
\hline & Female & 1.05 & $0.84-1.30$ & 0.67 & & & \\
\hline $\mathrm{CEA} \mathrm{level}^{\mathrm{a}}$ & & 1.001 & $1.0001-1.0002$ & 0.0001 & 1.0001 & {$[1.0000 ; 1.0002]$} & 0.0002 \\
\hline \multirow[t]{2}{*}{ WHO PS } & $0-1$ & 1 & & & & & \\
\hline & $\geq 2$ & 2.75 & $1.97-3.85$ & 0.001 & & & \\
\hline \multirow[t]{2}{*}{ B-Raf status } & Wild type & 1 & & & & & \\
\hline & Mutated & 2.29 & $1.19-4.40$ & 0.013 & & & \\
\hline \multirow[t]{2}{*}{ K-Ras status } & Wild type & 1 & & & & & \\
\hline & Mutated & 1.42 & $0.98-2.03$ & 0.06 & & & \\
\hline \multirow[t]{2}{*}{ Evolution } & Metachronous & 1 & & & & & \\
\hline & Synchronous & 1.35 & $1.08-1.7$ & 0.01 & & & \\
\hline \multirow[t]{2}{*}{ Primary tumor resection } & No & 1 & & & 1 & & \\
\hline & Yes & 0.32 & $0.24-0.43$ & $<\mathbf{0 . 0 0 0 1}$ & 0.36 & {$[0.26 ; 0.49]$} & $<\mathbf{0 . 0 0 0 1}$ \\
\hline \multirow[t]{2}{*}{ Complete surgery of metastases } & Yes & 1 & & & 1 & & \\
\hline & No & 2.56 & $2-3.45$ & $<\mathbf{0 . 0 0 0 1}$ & 0.48 & {$[0.34 ; 0.67]$} & $<\mathbf{0 . 0 0 0 1}$ \\
\hline \multirow[t]{2}{*}{ Localization of primary tumor } & Rectum & 1 & & & & & \\
\hline & Colon & 1.33 & $1.01-1.75$ & 0.04 & & & \\
\hline \multirow[t]{2}{*}{ Anti-EGFR therapy } & Yes & 1 & & & 1 & & \\
\hline & No & 1.35 & $1.09-1.69$ & 0.008 & 0.80 & {$[0.61 ; 1.04]$} & 0.1 \\
\hline \multirow[t]{2}{*}{ No. of metastatic sites } & 1 & 1 & & & 1 & & \\
\hline & $>1$ & 1.30 & $1.11-1.51$ & 0.012 & 1.05 & {$[0.88 ; 1.27]$} & $\mathbf{0 . 5 5}$ \\
\hline \multirow[t]{2}{*}{ Bevacizumab use } & No & 1 & & & 1 & & \\
\hline & Yes & 0.61 & $0.49-0.77$ & $<0.0001$ & 0.64 & {$[0.50 ; 0.81]$} & 0.0007 \\
\hline
\end{tabular}

${ }^{\mathrm{a}}$ Hazard ratio for continuous variable was calculated for 1 unit

$H R$ hazard ratio, $C I$ confidence interval, CEA carcinoembryonic antigen, WHO World Health Organization, PS performance status, EGFR epidermal growth factor receptor

prognostic factor of good outcome only in the group of patients with primary tumor resected (data not shown).

Interestingly, in patients who underwent primary tumor resection, we also observed that bevacizumab use was significantly associated with improved OS both in patients with metachronous metastases (HR 0.45, $95 \%$ CI $0.30-0.68, P<0.0001$ ) (median OS 49.3 months when treated with bevacizumab vs. 25.6 months when treated with chemotherapy alone) (Fig. 2c) and in patients with synchronous metastases (HR $0.74,95 \%$ CI $0.50-0.98$, $P=0.04$ ) (median OS 41 months when treated with bevacizumab vs. 24.8 months when treated with chemotherapy alone) (Fig. 2d). Again, such significant differences in survival were not observed in nonresected patients (Fig. 2e). Interestingly, subgroup analysis emphasized that bevacizumab used as a first-line therapy or after first-line therapy improved survival of patients with primary tumor resection $(P=0.01$ and $P=0.02$, respectively) but not patients without primary tumor resection.
Effect of Primary Tumor Resection and Bevacizumab Use on OS in the Validation Cohort $(n=328)$

As in the study cohort, survival association of bevacizumab was assessed in patients who initially underwent primary tumor resection $(n=232)$ and in nonresected patients $(n=96)$. Both groups of patients with or without primary tumor resection were comparable for the available classical prognostic factors (Supplementary Table S2). As in the study cohort, bevacizumab improved OS in the whole population (median OS 23 months when treated with bevacizumab vs. 16 months when treated with chemotherapy alone) (HR $0.67,95 \%$ CI $0.52-0.85$, $P=0.0008$ ) (Supplementary Fig. S1A). The effect of bevacizumab on OS was significant only in patients who underwent primary tumor resection before beginning treatment for mCRC. In these patients with primary tumor resection, median OS was 27 months in the bevacizumab group and 16 months in the chemotherapy-alone group 
TABLE 3 Patient and tumor characteristics $(n=409)$

\begin{tabular}{|c|c|c|c|c|c|}
\hline Characteristic & Variable & $\begin{array}{l}\text { No. resection of primary } \\
\text { tumor }(n=76)\end{array}$ & $\begin{array}{l}\text { Resection of primary } \\
\text { tumor }(n=333)\end{array}$ & Overall $(n=409)$ & $\begin{array}{l}P \text { value, } \\
\chi^{2} \text { test }\end{array}$ \\
\hline \multirow[t]{2}{*}{ Age, (year) } & Median [min; max] & $65.1[35 ; 86]$ & $65.5[24 ; 90]$ & $65.5[24 ; 90]$ & 0.98 \\
\hline & Mean (SD) & $66.2(10.3)$ & $64(11.5)$ & $65(11)$ & \\
\hline \multirow[t]{2}{*}{ Sex } & Male & $37(48.5 \%)$ & $183(55 \%)$ & $220(53.5 \%)$ & 0.33 \\
\hline & Female & $39(51.5 \%)$ & $150(45 \%)$ & $189(46.5 \%)$ & \\
\hline Death & & 65 & 251 & 312 & 0.04 \\
\hline \multirow[t]{3}{*}{ WHO PS } & $0-1$ & $48(63 \%)$ & $194(58 \%)$ & $242(59 \%)$ & 0.12 \\
\hline & $2-3$ & $14(18.5 \%)$ & $30(9 \%)$ & $44(11 \%)$ & \\
\hline & Unknown & $14(18.5 \%)$ & $109(33 \%)$ & $123(30 \%)$ & \\
\hline \multirow[t]{3}{*}{ B-Raf status } & Wild type & $15(20 \%)$ & $91(27.5 \%)$ & $106(26 \%)$ & 0.19 \\
\hline & Mutated & $4(5 \%)$ & $8(2.5 \%)$ & $12(3 \%)$ & \\
\hline & Unknown & $57(75 \%)$ & $234(70 \%)$ & $291(71 \%)$ & \\
\hline \multirow[t]{3}{*}{ K-Ras status } & Wild type & $17(22 \%)$ & $96(29 \%)$ & $113(27 \%)$ & 0.95 \\
\hline & Mutated & $14(18 \%)$ & $75(22.5 \%)$ & $89(22 \%)$ & \\
\hline & Unknown & $45(60 \%)$ & $162(48.5 \%)$ & $207(51 \%)$ & \\
\hline \multirow[t]{2}{*}{ Evolution } & Synchronous & $76(100 \%)$ & $179(54 \%)$ & $254(62 \%)$ & $<\mathbf{0 . 0 0 0 1}$ \\
\hline & Metachronous & $0(0 \%)$ & $153(46 \%)$ & $153(38 \%)$ & \\
\hline \multirow[t]{2}{*}{ Complete surgery of metastases } & No & $56(73.5 \%)$ & $251(75 \%)$ & $307(74 \%)$ & 0.87 \\
\hline & Yes & $20(26.5 \%)$ & $82(25 \%)$ & $102(26 \%)$ & \\
\hline \multirow[t]{3}{*}{ Localization of the primary tumor } & Colon & $59(78 \%)$ & $262(78.5 \%)$ & $320(78 \%)$ & 0.87 \\
\hline & Rectum & $16(22 \%)$ & $71(20.5 \%)$ & $87(21.5 \%)$ & \\
\hline & Unknown & 0 & $2(1 \%)$ & $2(0.5 \%)$ & \\
\hline \multirow[t]{2}{*}{ EGFR therapy } & Yes & $30(39.5 \%)$ & $137(41 \%)$ & $167(41 \%)$ & 0.65 \\
\hline & No & $46(40.5 \%)$ & $196(59 \%)$ & $242(59 \%)$ & \\
\hline \multirow[t]{2}{*}{ Bevacizumab use } & Yes & $36(47 \%)$ & $139(42 \%)$ & $175(43 \%)$ & 0.54 \\
\hline & No & $40(53 \%)$ & $194(58 \%)$ & $234(57 \%)$ & \\
\hline \multirow[t]{3}{*}{ No. of treatment lines } & 1 & $15(20 \%)$ & $36(11 \%)$ & $51(12.5 \%)$ & 0.01 \\
\hline & 2 & $19(25 \%)$ & $52(16 \%)$ & $71(17 \%)$ & \\
\hline & 3 or more & $42(55 \%)$ & $245(73 \%)$ & $287(70.5 \%)$ & \\
\hline \multirow[t]{2}{*}{ Metastases involving sites } & 1 & $40(53 \%)$ & $208(62 \%)$ & $248(60 \%)$ & 0.15 \\
\hline & $>1$ & $36(47 \%)$ & $125(38 \%)$ & $161(40 \%)$ & \\
\hline \multirow[t]{2}{*}{ CEA level } & Median [min; max] & $141[0 ; 14660]$ & $14[0 ; 20800]$ & $18[0 ; 20800]$ & 0.17 \\
\hline & Mean (SD) & 747 (2160) & 370 (1902) & 447 (1961) & \\
\hline
\end{tabular}

WHO World Health Organization, $P S$ performance status, EGFR epidermal growth factor receptor, CEA carcinoembryonic antigen

(HR 0.65, $95 \%$ CI 0.49-0.88, log-rank test $P=0.0035$ ) (Supplementary Fig. S1B). In contrast, bevacizumab use did not affect OS in nonresected patients. In these patients, median OS was 20 months in the bevacizumab group and 17 months in the chemotherapy-alone group (HR 0.73, $95 \%$ CI $0.48-1.12$, log-rank test $P=0.15$ ) (Supplementary Fig. S1C).

\section{DISCUSSION}

This retrospective study demonstrated that the improved survival associated with the addition of adding bevacizumab to fluoropyrimidine-based chemotherapy appears to be dependent on the presence of the primary tumor in mCRC patients.

In $\mathrm{mCRC}$ patients, the role of resection of the primary tumor remains unclear. Case reports suggested that resection of the primary tumor could increase the growth rate of liver metastases on increased vascular density, proliferation rate, and metabolic growth rate. ${ }^{17-19}$ Taken together, these data suggest that the behavior of metastatic disease could be dependent on the primary tumor. Interestingly, several retrospective clinical studies have reported improved outcome of patients with $\mathrm{mCRC}$ who underwent primary tumor resection compared to nonresected patients. ${ }^{20-22}$ Unfortunately, the use of systemic therapy has not been recorded in some of these studies. 
FIG. 2 Kaplan-Meier curves for OS according to treatment: chemotherapy with bevacizumab (bevacizumab group), or chemotherapy without bevacizumab (chemotherapy-alone group), in patients who previously underwent primary tumor resection $(n=333)$ a and in patients who did not $(n=76)$ b. Kaplan-Meier curves for OS according to treatment: chemotherapy with bevacizumab (bevacizumab group), or chemotherapy without bevacizumab (chemotherapy-alone group), in patients with synchronous metastatic disease who previously underwent primary tumor resection $(n=153) \mathbf{c}$ and in patients with synchronous metastatic disease and without primary tumor resection $(n=76) \mathbf{d}$ and in patients with metachronous metastatic disease $(n=180)$ e. $P$ values were calculated by log-rank test

\section{a}

\section{Survival}

probability (\%) Logrank test $\mathrm{P}=0.0003$

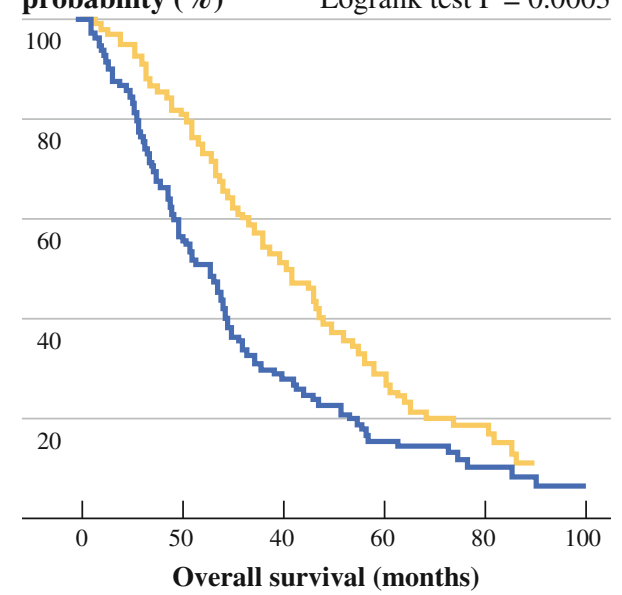

c

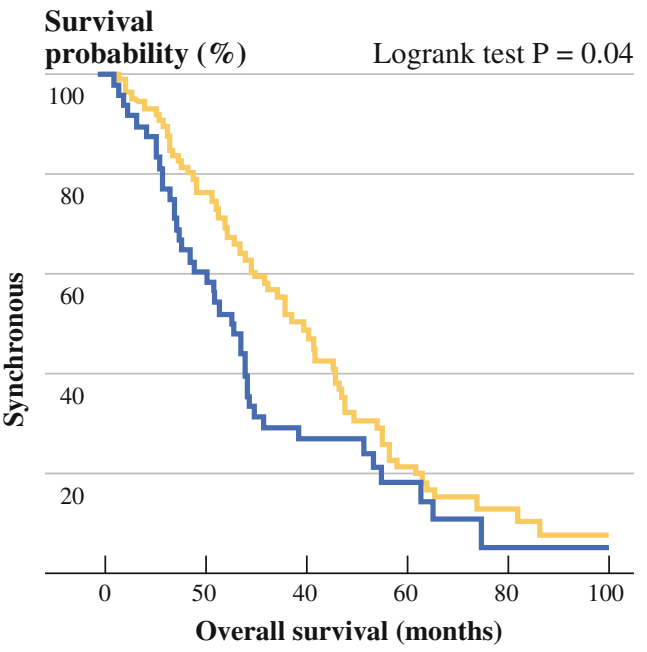

e

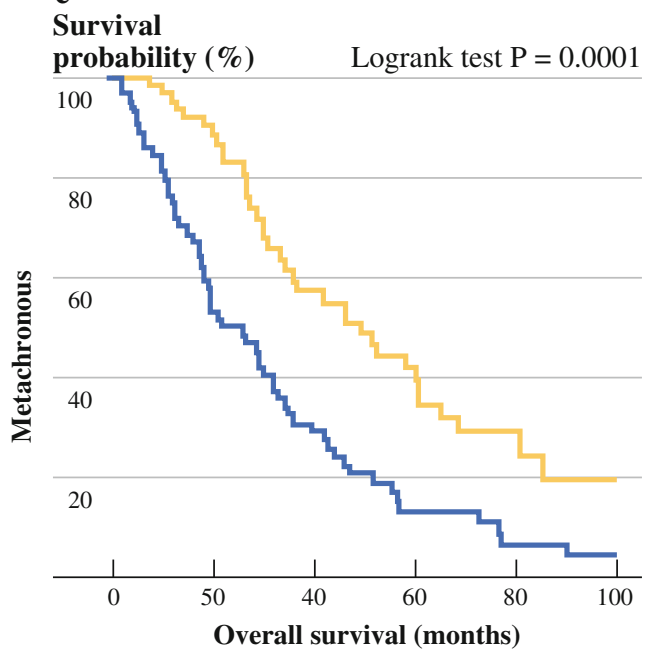

b

Survival

probability (\%) Logrank test $\mathrm{P}=0.49$

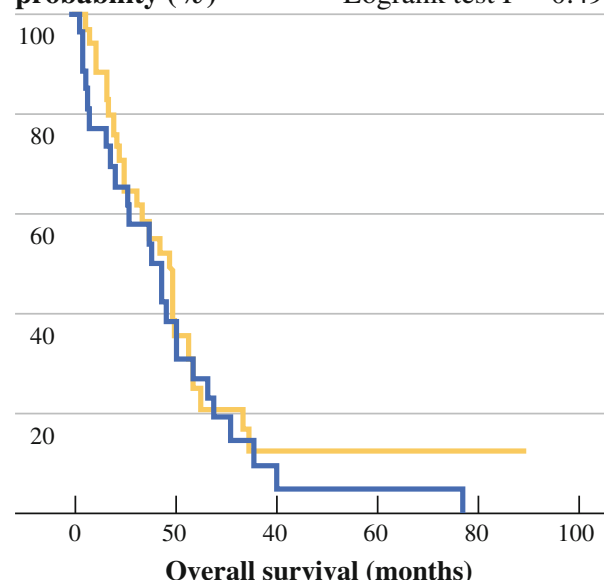

d

Survival

probability (\%) Logrank test $\mathrm{P}=0.49$

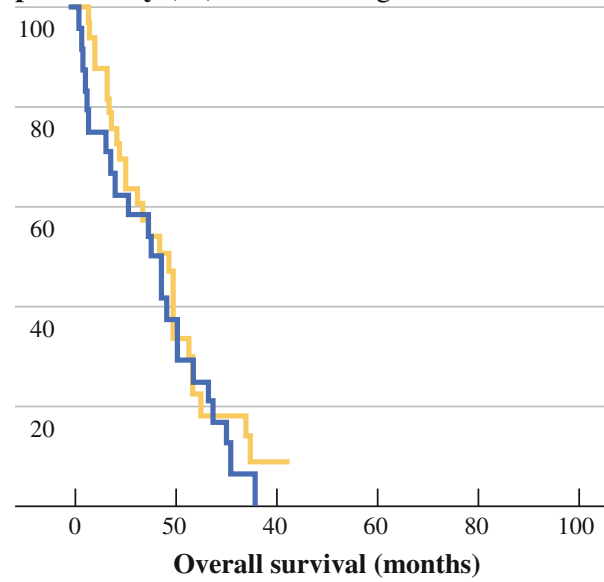

Overall survival (months)
- Chemotherapy alone Bevacizumab
A first hypothesis to explain the detrimental effect of primary colorectal cancers on bevacizumab efficacy is the presence of metastatic dormancy imposed by the primary tumor. However, our data indicate that the presence of the primary tumor is correlated to a shorter OS, even in the absence of bevacizumab, ruling out the possibility that 
primary colon cancers reduce the kinetic of metastatic growth. This observation is supported by a retrospective analysis of two phase III clinical trials (CAIRO and CAIRO2). This study also underlined the favorable prognostic value of primary tumor resection in mCRC patients in terms of OS improvement. ${ }^{20}$

Preclinical studies raised caution on the antitumoral effects of antiangiogenic agents when primary tumor is in place. Indeed, in some animal models, angiogenesis inhibition can alter the natural history of tumors by increasing invasion and metastasis. In mCRC, the proangiogenic network in the tumor microenvironment could be different between the primary tumor and its related metastases. Indeed, it has been reported that VEGF expression is higher in colorectal cancer metastases than in the primary tumor. ${ }^{23}$ Moreover, colorectal cancer cell lines isolated from primary tumor were characterized by a higher expression of multiple proangiogenic factors, such as placental growth factor, thrombopoietin, and transforming growth factor $\beta 1$, compared to tumor cell lines isolated from metastases. ${ }^{14}$ Taken together, these data are consistent with a more complex tumor microenvironment angiogenic network in the primary tumor than in the metastases. Thus, because metastases may be more dependent on VEGF-driven angiogenesis, targeting VEGF with bevacizumab may be more effective on metastases than on the primary tumor.

Moreover, in experimental tumor models, VEGF inhibition may also promote tumor invasion and could enhance metastasis. ${ }^{24}$ In these experiments, despite an initial antitumor benefit, antiangiogenic therapies may facilitate induction of invasive and metastatic tumor outgrowths. This in turn could limit the overall benefits in terms of OS. Ebos et al. ${ }^{25}$ observed in other animal models that although VEGFR inhibitor could delay the growth of orthotopically implanted tumors, such treatment also induced accelerated metastatic dissemination. Together, such data provide a biological rationale for the clinical results observed in our study, suggesting that systemic reactions to VEGF inhibition could facilitate tumor dissemination and may impede bevacizumab efficacy when primary tumor stays in place.

Limitations of our study include its two-center patient recruitment and its retrospective design. However, the use of two large independent cohorts, the homogeneity of results between both cohorts, and their separate analysis strongly confirm our original observation.

Moreover, the reason behind the decision of primary tumor resection had not been recorded, so we cannot exclude a confounding factor. In addition, the separation of patients into four groups reduces the number of patients per group and may impact on outcome. However, a comparison of patients who underwent resection of the primary tumor with unresected patients did not reveal in any differences in classical prognosis factors.
In conclusion, our results raised the possibility that bevacizumab is associated with improvement in OS only in patients with primary tumor resection, and thus our findings indicate a possible new predictive marker of bevacizumab efficacy. On the other hand, it seems that patients without primary tumor resection obtain no survival advantage from bevacizumab use. Such information is important and may reduce health care costs and toxic events in the subgroup of patients with primary tumor in place. Moreover, our results could explain, at least in part, conflicting results among clinical trials concerning bevacizumab efficacy in mCRC patients, underscoring the need to report the proportion of patients with primary tumor resection.

These data support the rationale to prospectively validate the influence of primary tumor resection on bevacizumab efficacy in synchronous stage IV colorectal cancer.

DISCLOSURE The authors declare no conflict of interest.

OPEN ACCESS This article is distributed under the terms of the Creative Commons Attribution License which permits any use, distribution, and reproduction in any medium, provided the original author(s) and the source are credited.

\section{REFERENCES}

1. Golfinopoulos V, Salanti G, Pavlidis N, et al. Survival and disease-progression benefits with treatment regimens for advanced colorectal cancer: a meta-analysis. Lancet Oncol. 2007;8: 898-911.

2. Kopetz S, Chang GJ, Overman MJ, et al. Improved survival in metastatic colorectal cancer is associated with adoption of hepatic resection and improved chemotherapy. J Clin Oncol. 2009;27: 3677-83.

3. O'Connell JB, Maggard MA, Ko CY, et al. Colon cancer survival rates with the new American Joint Committee on Cancer sixth edition staging. J Natl Cancer Inst. 2004;96:1420-5.

4. Sanoff HK, Sargent DJ, Campbell ME, et al. Five-year data and prognostic factor analysis of oxaliplatin and irinotecan combinations for advanced colorectal cancer: N9741. J Clin Oncol. 2008;26:5721-7.

5. Chua TC, Saxena A, Liauw W, et al. Hepatectomy and resection of concomitant extrahepatic disease for colorectal liver metastases-a systematic review. Eur J Cancer. 2012;48:1757-65.

6. Fuchs CS, Marshall J, Mitchell E, et al. Randomized, controlled trial of irinotecan plus infusional, bolus, or oral fluoropyrimidines in first-line treatment of metastatic colorectal cancer: results from the BICC-C study. J Clin Oncol. 2007;25:4779-86.

7. Tabernero J, Van Cutsem E, Diaz-Rubio E, et al. Phase II trial of cetuximab in combination with fluorouracil, leucovorin, and oxaliplatin in the first-line treatment of metastatic colorectal cancer. J Clin Oncol. 2007;25:5225-32.

8. Saltz LB, Clarke S, Diaz-Rubio E, et al. Bevacizumab in combination with oxaliplatin-based chemotherapy as first-line therapy in metastatic colorectal cancer: a randomized phase III study. $J$ Clin Oncol. 2008;26:2013-9.

9. Bennouna J, Sastre J, Arnold D, et al. Continuation of bevacizumab after first progression in metastatic colorectal cancer 
(ML18147): a randomised phase 3 trial. Lancet Oncol. 2013;14:29-37.

10. Hurwitz H, Fehrenbacher L, Novotny W, et al. Bevacizumab plus irinotecan, fluorouracil, and leucovorin for metastatic colorectal cancer. N Engl J Med. 2004;350:2335-42.

11. Giantonio BJ, Catalano PJ, Meropol NJ, et al. Bevacizumab in combination with oxaliplatin, fluorouracil, and leucovorin (FOLFOX4) for previously treated metastatic colorectal cancer: results from the Eastern Cooperative Oncology Group Study E3200. J Clin Oncol. 2007;25:1539-44.

12. Van Cutsem E, Rivera F, Berry S, et al. Safety and efficacy of first-line bevacizumab with FOLFOX, XELOX, FOLFIRI and fluoropyrimidines in metastatic colorectal cancer: the BEAT study. Ann Oncol. 2009;20:1842-7.

13. Grothey A, Sugrue MM, Purdie DM, et al. Bevacizumab beyond first progression is associated with prolonged overall survival in metastatic colorectal cancer: results from a large observational cohort study (BRiTE). J Clin Oncol. 2008;26:5326-34.

14. Abajo A, Bitarte N, Zarate R, et al. Identification of colorectal cancer metastasis markers by an angiogenesis-related cytokineantibody array. World J Gastroenterol. 2012;18:637-45.

15. Ebos JM, Kerbel RS. Antiangiogenic therapy: impact on invasion, disease progression, and metastasis. Nat Rev Clin Oncol. $2011 ; 8: 210-21$.

16. Levesque LE, Hanley JA, Kezouh A, et al. Problem of immortal time bias in cohort studies: example using statins for preventing progression of diabetes. BMJ. 2010;340:b5087.

17. Peeters CF, Westphal JR, de Waal RM, et al. Vascular density in colorectal liver metastases increases after removal of the primary tumor in human cancer patients. Int J Cancer. 2004;112:554-9.
18. Peeters CF, de Waal RM, Wobbes T, et al. Outgrowth of human liver metastases after resection of the primary colorectal tumor: a shift in the balance between apoptosis and proliferation. Int $J$ Cancer. 2006;119:1249-53.

19. Scheer MG, Stollman TH, Vogel WV, et al. Increased metabolic activity of indolent liver metastases after resection of a primary colorectal tumor. J Nucl Med. 2008;49:887-91.

20. Venderbosch S, de Wilt JH, Teerenstra S, et al. Prognostic value of resection of primary tumor in patients with stage IV colorectal cancer: retrospective analysis of two randomized studies and a review of the literature. Ann Surg Oncol. 2011;18:3252-60.

21. Stillwell AP, Buettner PG, Ho YH, et al. Meta-analysis of survival of patients with stage IV colorectal cancer managed with surgical resection versus chemotherapy alone. World J Surg. 2010;34:797-807.

22. Karoui M, Roudot-Thoraval F, Mesli F, et al. Primary colectomy in patients with stage IV colon cancer and unresectable distant metastases improves overall survival: results of a multicentric study. Dis Colon Rectum. 2011;54:930-8.

23. Kim YW, Ko YT, Kim NK, et al. A comparative study of protein expression in primary colorectal cancer and synchronous hepatic metastases: the significance of matrix metalloproteinase-1 expression as a predictor of liver metastasis. Scand J Gastroenterol. 2010;45:217-25.

24. Paez-Ribes M, Allen E, Hudock J, et al. Antiangiogenic therapy elicits malignant progression of tumors to increased local invasion and distant metastasis. Cancer Cell. 2009;15:220-31.

25. Ebos JM, Lee CR, Cruz-Munoz W, et al. Accelerated metastasis after short-term treatment with a potent inhibitor of tumor angiogenesis. Cancer Cell. 2009;15:232-9. 